\title{
Adaptabilidade e estabilidade em feijão-vagem de crescimento indeterminado
}

\author{
Nei Peixoto ${ }^{1}$; Leila T. Braz ${ }^{2}$; David A. Banzatto ${ }^{2}$; Ademar P. Oliveira ${ }^{3}$ \\ ${ }^{1}$ AGENCIARURAL, EE Anápolis, C. Postal 608, 75.001-970 Anápolis-GO; ${ }^{2}$ UNESP-FCAV Depto. Produção Vegetal, 14.870-000 \\ Jaboticabal-SP; ${ }^{3}$ UFPB -CCA Depto. Fitotecnia, C. Postal 02, 58.397-000 Areia-PB.
}

\section{RESUMO}

Estudaram-se a adaptabilidade e estabilidade de 15 genótipos de feijão-vagem de crescimento indeterminado em relação a oito ambientes. Houve diferenças significativas entre genótipos em apenas três ambientes. As linhagens Hav 13, Hav 14, Hav 53 e Hav 56 igualaram-se aos genótipos mais produtivos em todos ambientes. As linhagens Hav 13, Hav 21, Hav 25, Hav 38, Hav 40, Hav 56, Hav 65 e a cultivar Favorito Ag 480 foram estáveis e as demais imprevisíveis, de acordo com a metodologia de Eberhart \& Russell. Os genótipos apresentaram ampla adaptabilidade, exceto Hav 67 adaptável a ambientes desfavoráveis. A linhagem Hav 13, de vagens cilíndricas e sementes brancas, foi a alternativa mais vantajosa à cultivar Favorito Ag 480, utilizada como testemunha.

Palavras-chave: Phaseolus vulgaris, genótipos, ambiente, produtividade.

\section{ABSTRACT}

\section{Adaptability and stability in pole beans}

The adaptability and stability of fifteen pole beans genotypes were evaluated in relation to eight environments. There were statistical differences among genotypes only in three environments. The breeding lines Hav 13, Hav 14, Hav 53 and Hav 56 were among the higher yielding genotypes in all environments. The breeding lines Hav 13, Hav 21, Hav 25, Hav 38, Hav 40, Hav 56, Hav 65 and the cultivar Favorito Ag 480 were stable, while the other ones were unforeseeable according to the Eberhart \& Russell method. Genotypes showed a wide adaptation in relation to yield of marketable pods, except Hav 67 adapted to unfavorable environments. The breeding line Hav 13, with cylindrical pods and white seeds was the most advantageous alternative to the standard cultivar Favorito Ag 480.

Keywords: Phaseolus vulgaris, genotypes, environment, yield.

(Recebido para publicação em 16 de maio de 2001 e aceito em 03 de julho de 2002)

A cultura de feijão-vagem no Brasil é conduzida tradicionalmente por produtores familiares, utilizando-se pequeno número de cultivares de crescimento indeterminado no sistema tutorado (Peixoto et al., 1993). Tais cultivares estão sendo utilizadas em diversas regiões, sem se considerar as possíveis diferenças de comportamento nos diversos ambientes.

A expressão fenotípica de caracteres variáveis depende da carga genética da cultivar, bem como do ambiente onde é plantada. Algumas cultivares podem apresentar produções estáveis, altas ou baixas, em uma ampla faixa de ambientes, enquanto outras apresentam variações, à medida que as condições ambientais são modificadas (Comstock \& Moll, 1963). Assim, o estudo da interação genótipos $\mathrm{x}$ ambientes tornase necessário nos programas de melhoramento, desde a escolha de progenitores à indicação e liberação de novas cultivares (Finlay \& Wilkinson, 1963; Eberhart \& Russell, 1966; Banzatto, 1994; Cruz \& Regazzi, 1994).
As empresas produtoras de sementes estão interessadas na obtenção de cultivares estáveis, que possam ser cultivadas em diferentes ambientes, enquanto que para o produtor seria desejável a utilização de cultivares adaptadas às suas condições edafoclimáticas $\mathrm{e}$ à tecnologia específica de produção.

Diversos métodos visando estudar a interação genótipo $\mathrm{x}$ ambiente têm sido propostos, destacando-se aqueles que se baseiam nas análises de variância, na regressão linear e na regressão segmentada (Duarte, 1988; Cruz \& Regazzi, 1994). Atualmente, os métodos de Finlay \& Wilkinson e de Eberhart \& Russell são os mais utilizados para o estudo de adaptabilidade e estabilidade, ambos eficientes para descrever o comportamento dos genótipos frente às variações ambientais, sendo que o segundo método, por utilizar escala aritmética, facilita a interpretação biológica dos resultados (Duarte 1988).

Este trabalho teve como objetivo avaliar, em cultura tutorada, a adaptabilidade e estabilidade de linhagens pro- missoras de feijão-vagem, comparadas

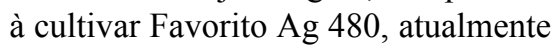
mais plantada no Brasil.

\section{MATERIAL E MÉTODOS}

Estudaram-se, em cultura tutorada, a adaptabilidade e estabilidade, de feijão vagem de crescimento indeterminado, envolvendo quatorze linhagens (Hav 13, Hav 14, Hav 21, Hav 22, Hav 25, Hav 38, Hav 40, Hav 41, Hav 49, Hav 53, Hav 56, Hav 65, Hav 67 e Hav 68), selecionadas a partir de material genético introduzido do Centro Internacional de Agricultura Tropical (CIAT) e a cultivar Favorito Ag 480, produzida pela Horticeres. Esses genótipos foram avaliados em oito ambientes (Anápolis-GO 1996, MorrinhosGO 1996, Urutaí-GO 1996, Anápolis 1996/97, Anápolis 1997, Jaboticabal-SP 1997, Anápolis 1998 e Areia-PB 1999). Os experimentos foram conduzidos no período de outono/inverno, exceto no ambiente Anápolis 1996/97, conduzido no período de primavera/verão. 
Tabela 1. Produtividade de vagens comerciáveis (t/ha) de quinze genótipos de feijão-vagem em oito ambientes. Anápolis, AGENCIARURAL, 1996-1999.

\begin{tabular}{|c|c|c|c|c|c|c|c|c|}
\hline \multirow{3}{*}{ Genótipos } & \multicolumn{8}{|c|}{ Locais/anos } \\
\hline & $\begin{array}{c}\text { Anápolis } \\
1996\end{array}$ & $\begin{array}{c}\text { Morrinhos } \\
1996\end{array}$ & $\begin{array}{c}\text { Urutaí } \\
1996\end{array}$ & $\begin{array}{c}\text { Anápolis } \\
1997\end{array}$ & $\begin{array}{c}\text { Anápolis } \\
1996 / 97\end{array}$ & $\begin{array}{l}\text { Jabotica- } \\
\text { bal } 1997\end{array}$ & $\begin{array}{c}\text { Anápolis } \\
1998\end{array}$ & Areia 1999 \\
\hline & \multicolumn{8}{|c|}{ Produtividade (t/ha) } \\
\hline Hav 13 & $12,83 a^{1}$ & $12,30 a$ & $15,83 a b c$ & $15,26 \mathrm{a}$ & $9,08 \mathrm{a}$ & $24,27 a$ & $23,03 a b$ & $25,58 \mathrm{a}$ \\
\hline Hav 14 & $10,89 a$ & $14,56 a$ & $14,13 \mathrm{abc}$ & $11,85 \mathrm{a}$ & $10,03 a$ & $21,24 a b$ & $22,20 a b$ & $20,79 a$ \\
\hline Hav 21 & $12,83 \mathrm{a}$ & $13,12 a$ & $18,13 a b$ & $13,78 \mathrm{a}$ & $9,41 \mathrm{a}$ & $19,05 \mathrm{bc}$ & $18,16 \mathrm{bc}$ & $27,63 \mathrm{a}$ \\
\hline Hav 22 & $11,75 a$ & $12,78 a$ & $14,32 \mathrm{abc}$ & $17,17 \mathrm{a}$ & $10,76 \mathrm{a}$ & $12,70 \mathrm{e}$ & $19,31 \mathrm{bc}$ & $31,60 \mathrm{a}$ \\
\hline Hav 25 & 13,17 a & $10,92 a$ & $12,38 \mathrm{bc}$ & $17,64 a$ & 9,44 a & $14,27 \mathrm{de}$ & $20,03 \mathrm{bc}$ & $26,51 \mathrm{a}$ \\
\hline Hav 38 & $12,14 a$ & $14,09 a$ & $15,96 a b c$ & $16,77 \mathrm{a}$ & 8,16 a & $19,96 a b$ & $19,32 \mathrm{bc}$ & 35,03 a \\
\hline Hav 40 & 15,43 a & $14,40 a$ & $19,83 \mathrm{a}$ & $14,21 \mathrm{a}$ & 9,37 a & $18,39 \mathrm{bcd}$ & 19,02 bc & 24,19 a \\
\hline Hav 41 & 12,83 a & $17,43 a$ & $9,13 \mathrm{c}$ & $11,46 \mathrm{a}$ & $10,49 a$ & $12,83 \mathrm{e}$ & $15,53 \mathrm{c}$ & 31,58 a \\
\hline Hav 49 & $12,63 \mathrm{a}$ & $13,68 a$ & $9,38 \mathrm{c}$ & $17,31 \mathrm{a}$ & $10,06 \mathrm{a}$ & $19,93 a b$ & $20,79 \mathrm{bc}$ & 35,74 a \\
\hline Hav 53 & $13,04 a$ & $12,06 a$ & $16,13 a b c$ & 17,76 a & $8,09 a$ & $21,90 a b$ & $21,99 a b$ & 20,86 a \\
\hline Hav 56 & $12,61 \mathrm{a}$ & $11,09 a$ & $14,88 \mathrm{abc}$ & $15,71 \mathrm{a}$ & $9,43 \mathrm{a}$ & $20,11 a b$ & $21,85 a b c$ & $22,68 \mathrm{a}$ \\
\hline Hav 65 & $14,12 \mathrm{a}$ & $12,62 a$ & $13,97 \mathrm{abc}$ & $14,20 \mathrm{a}$ & $8,94 \mathrm{a}$ & $22,30 a b$ & $19,84 \mathrm{bc}$ & 20,47 a \\
\hline Hav 67 & 13,17 a & $12,86 a$ & $13,83 a b c$ & $16,83 a$ & $9,42 a$ & $19,81 a b$ & $20,51 \mathrm{bc}$ & 15,92 a \\
\hline Hav 68 & $12,36 \mathrm{a}$ & $13,28 a$ & $9,08 \mathrm{c}$ & $15,25 \mathrm{a}$ & $10,15 a$ & $20,78 a b$ & $27,60 \mathrm{a}$ & $30,50 \mathrm{a}$ \\
\hline $\begin{array}{l}\text { Favorito } \mathrm{Ag} \\
480\end{array}$ & $13,42 \mathrm{a}$ & $15,27 a$ & $14,50 \mathrm{abc}$ & $16,30 \mathrm{a}$ & $10,49 a$ & $14,97 \mathrm{cde}$ & $24,20 a b$ & 30,28 a \\
\hline Média & $12,88 \mathrm{DE}^{2}$ & $13,36 \mathrm{D}$ & $14,08 \mathrm{D}$ & $15,30 \mathrm{CD}$ & $9,55 \mathrm{E}$ & $18,84 \mathrm{BC}$ & $20,89 \mathrm{~B}$ & $26,62 \mathrm{~A}$ \\
\hline CV (\%) & 12,63 & 17,50 & 16,95 & 13,38 & 16,83 & 8,38 & 10,06 & 25,02 \\
\hline
\end{tabular}

Médias seguidas da mesma letra minúscula, na coluna ${ }^{1}$, e maiúscula na linha², não diferem entre si pelo teste de Tukey $(\mathrm{P}>0,05)$.

As adubações de plantio e de cobertura foram realizadas, conforme resultados da análise de solo e as recomendações para cada local. Foram realizados controles fitossanitários e os tratos culturais normais para a cultura, incluindo irrigação por aspersão, procurando-se manter o nível de disponibilidade de água acima de $80 \%$ da capacidade de campo.

O delineamento experimental utilizado foi em blocos casualizados com três repetições. As parcelas foram constituídas de 20 plantas, dispostas em duas fileiras, espaçadas de um metro, ocupando área de $4 \mathrm{~m}^{2}$, todas úteis, exceto em Areia (PB), onde apenas 10 plantas foram consideradas. $\mathrm{O}$ espaçamento entre covas na fileira foi de $20 \mathrm{~cm}$, com uma planta por cova, exceto em Jaboticabal (SP), onde foi de $40 \mathrm{~cm}$, com duas plantas por cova.

Os dados de produtividade de vagens comerciáveis foram submetidos à análise de interação genótipo $\mathrm{x}$ ambiente pelo método de Eberhart \& Russell
(1966), utilizando-se o programa IGA, desenvolvido por Banzatto (1994). Este programa realiza a análise conjunta dos dados dos diversos ambientes dentro de cada grupo de ensaios e, dentro de cada ambiente, a análise de variância e teste de médias.

A metodologia de Eberhart \& Russell (1966) baseia-se numa equação de regressão linear, representada pelo modelo matemático:

$$
\begin{aligned}
Y_{i j}=m_{i}+b_{i} I_{j}+d_{i j}+e_{i j ~}=1,2, \ldots, I \\
j=1,2, \ldots, J
\end{aligned}
$$

no qual ${ }_{i}$ representa os genótipos, os ambientes, sendo os termos assim definidos: te $\mathrm{j}$;

$\mathrm{Y}_{\mathrm{ij}}$ média do genótipo i no ambien-

$\mathrm{m}_{\mathrm{i}}$ média do genótipo $\mathrm{i}$, considerando todos os ambientes;

$b_{\mathrm{i}}$ : coeficiente de regressão linear, que mede a resposta do i-ésino genótipo à variação ambiental (adaptabilidade);

$\mathrm{I}_{\mathrm{j}}$ : índice ambiental do j-ésimo ambiente, obtido pela diferença entre a média de todos os genótipos neste ambiente e a média geral de todos os genótipos em todos os ambientes;

$\mathrm{d}_{\mathrm{i} i}$ : desvio da regressão linear, do iésimo genótipo no j-ésimo ambiente;

mede a resposta do genótipo às flutuações que podem ocorrer nos ambientes (estabilidade).

\section{RESULTADOS E DISCUSSÃO}

Houve diferenças significativas entre genótipos em Urutaí 1996, Jaboticabal 1997 e Anápolis 1998. As linhagens Hav 13, Hav 14, Hav 53 e Hav 56 igualaram-se aos genótipos mais produtivos em todos os ambientes. Diferiram-se dos genótipos mais produtivos, as linhagens Hav 21, Hav 22 e Hav 40, em Jaboticabal 1997 e Anápolis 1998; Hav 25 e Hav 41, em Urutaí 1996, Jaboticabal 1997 e Anápolis 1998; Hav 49, em Urutaí 1996 e Anápolis 1998 (Tabela 1). As linhagens Hav 13, Hav 21, Hav 25, Hav 38, Hav 40, Hav 56, Hav 65 e a cultivar Favorito Ag 480 foram estáveis, como pode ser constatado 
Tabela 2. Desempenho de quinze genótipos de feijão-vagem quanto à produtividade média de vagens comerciáveis, em relação a oito ambientes. Anápolis, AGENCIARURAL, 1996-1999.

\begin{tabular}{|c|c|c|c|c|c|}
\hline \multirow[t]{2}{*}{ Genótipos } & \multirow{2}{*}{$\begin{array}{c}\begin{array}{c}\text { Produtividade } \\
\text { (t/ha) }\end{array} \\
\mathrm{mi}\end{array}$} & \multicolumn{2}{|c|}{ Coeficientes de regressão linear } & \multirow{2}{*}{$\begin{array}{c}\begin{array}{c}\text { Desvios de } \\
\text { regressão }\end{array} \\
\text { dij }\end{array}$} & \multirow{2}{*}{$\begin{array}{c}\begin{array}{c}\text { Coeficientes de } \\
\text { determinação }\end{array} \\
\text { R2i }\end{array}$} \\
\hline & & bi & $\mathbf{t}(\mathbf{b i}$ & & \\
\hline Hav 13 & $17,27 \mathrm{a}^{1}$ & 1,07 & $0,43 \mathrm{~ns}^{2}$ & $2,52 \mathrm{~ns}^{3}$ & $0,88 * *$ \\
\hline Hav 14 & 15,71 a & 0,79 & $-1,11 \mathrm{~ns}$ & 4,59 * & 0,74 ** \\
\hline Hav 21 & $16,51 \mathrm{a}$ & 0,96 & $-0,24$ ns & $1,49 \mathrm{~ns}$ & 0,88 ** \\
\hline Hav 22 & $16,30 \mathrm{a}$ & 1,12 & 0,49 ns & 8,43 ** & 0,79 ** \\
\hline Hav 25 & $15,55 \mathrm{a}$ & 0,97 & $-0,23$ ns & $1,89 \mathrm{~ns}$ & 0,87 ** \\
\hline Hav 38 & $17,68 \mathrm{a}$ & 1,41 & 2,45 ns & $2,92 \mathrm{~ns}$ & 0,92 ** \\
\hline Hav 40 & 16,86 a & 0,72 & $-1,69$ ns & $2,63 \mathrm{~ns}$ & 0,76 ** \\
\hline Hav 41 & 15,16 a & 1,05 & $0,14 \mathrm{~ns}$ & $19,10 * *$ & 0,63 * \\
\hline Hav 49 & $17,44 \mathrm{a}$ & 1,49 & 2,33 ns & $6,02 *$ & 0,89 ** \\
\hline Hav 53 & 16,22 a & 0,81 & $-0,99$ ns & 4,38 * & $0,75^{* *}$ \\
\hline Hav 56 & $16,04 \mathrm{a}$ & 0,87 & $-1,02$ ns & $0,26 \mathrm{~ns}$ & 0,89 ** \\
\hline Hav 65 & $15,78 a$ & 0,73 & $-1,55 \mathrm{~ns}$ & $3,11 \mathrm{~ns}$ & $0,75 * *$ \\
\hline Hav 67 & 15,29 a & 0,48 & $-2,58 *$ & 5,53 * & $0,48 \mathrm{~ns}$ \\
\hline Hav 68 & 17,37 a & 1,41 & 2,06 ns & 5,07 * & 0,89 ** \\
\hline Favorito $\mathrm{Ag} 480$ & $17,44 \mathrm{a}$ & 1,13 & $0,77 \mathrm{~ns}$ & $2,69 \mathrm{~ns}$ & $0,88^{* *}$ \\
\hline População & 16,44 & 1,00 & & & \\
\hline
\end{tabular}

${ }^{1}$ Médias seguidas da mesma letra, na coluna, não diferem entre si pelo teste de Tukey $(\mathrm{P}>0,05) ;{ }^{2} \mathrm{Teste} t ;{ }^{3}$ Teste $\mathrm{F}$.

Níveis de significância: $n s=$ não significativo $(\mathrm{P}>0,05) ; *=$ significativo $(\mathrm{P}>0,05) ; * *=$ significativo $(\mathrm{P}>0,01)$.

pela não significância do teste $\mathrm{F}$ para os desvios de regressão $\left(\mathrm{d}_{\mathrm{ij}} @ 0\right)$, enquanto que as demais mostraram-se imprevisíveis $\left(\mathrm{d}_{\mathrm{ij}}\right.$ ? 0$)$.

Os genótipos apresentaram ampla adaptabilidade (com b @1), exceto Hav 67, que revelou-se adaptável a ambientes desfavoráveis $\left(\mathrm{com} \mathrm{b}_{\mathrm{i}}<1\right)$. O modelo de regressão linear foi adequado, tendo em vistas a significância dos coeficientes de determinação $\left(\mathrm{R}^{2}\right)$, exceto para Hav 41 (Tabela 2).

$\mathrm{O}$ desvio da regressão linear $\mathrm{d}_{\mathrm{ij}}$ indica o grau de confiabilidade da resposta linear estimada. Um genótipo com baixo valor de $\mathrm{d}_{\mathrm{ij}}$ deverá apresentar comportamento uniforme, quando cultivado em condições ambientais semelhantes, enquanto que um genótipo com alto valor de $\mathrm{d}_{\mathrm{ij}}$ dificilmente repetirá esse desempenho. Adicionalmente, o coeficiente de determinação $\mathrm{R}^{2}$ mede a adequação do modelo. Uma cultivar ideal deverá apresentar alto rendimento médio $\left(\mathrm{m}_{\mathrm{i}}\right)$, resposta positiva à melhoria do ambiente, isto é com b@ $@ 1$ e o comportamento altamente previsível, ou seja $\mathrm{d}_{\mathrm{ij}} @ 0$ (Bonato, 1978), além do coeficiente de determinação $\mathrm{R}^{2}$ @ 1 (Banzatto, 1994).
As linhagens Hav 38, Hav 56 e Hav 65 , todas de vagens achatadas de sementes pretas, podem ser utilizadas como alternativas para o mercado, onde esse tipo de vagem é bem aceito. Vagens cilíndricas são preferidas na maioria dos mercados brasileiros, visto que vagens achatadas são associadas por consumidores ao feijão comum, de vagens fibrosas, o que nem sempre é verdadeiro, conforme demonstrado por Hamasaki et al. (1998). Já cultivares com sementes coloridas expressam essa cor após o preparo, tanto como conserva quanto para o consumo imediato, principalmente se as vagens forem colhidas no máximo tamanho comercial.

A linhagem Hav 13, de vagens cilíndricas e sementes brancas, revelouse a alternativa vantajosa à cultivar $\mathrm{Fa}$ vorito Ag 480, utilizada como testemunha, pois além de estável, igualou-se aos genótipos mais produtivos em todos os ambientes, o que não ocorreu com esta.

\section{LITERATURA CITADA}

BANZATTO, D.A. Comparação de métodos de avaliação da adaptabilidade e estabilidade de cultivares de batata. Jaboticabal: FCAV, 1994. 170 p. (Tese livre docência).
BONATO, E.R. Estabilidade fenotípica da produção de grãos de dez cultivares de soja (Glycine Max (L) Merril ) nas condições deo Rio Grande do Sul. Piracicaba, 1978, 123 p. ESALQ (Tese doutorado).

COMSTOCK, R.E., MOLL, R.H. Genotypeenvironment interactions. In: HANSON, W.D., ROBINSON, H.F. Eds. Statistical genetics and plant breeding, Washington: National Academy of Sciences - National Research Council, p. 15496, 1963. (Publication 982)

CRUZ, C.D.; REGAZZI, A.J. Modelos biométricos aplicados ao melhoramento de plantas. Viçosa. UFV, Imprensa Universitária, 1994. 330 p.

DUARTE, J.B. Estudo da adaptabilidade e estabilidade fenotipica em linhagens e cultivares de feijão mulatinho (Phaseolus vulgaris L.). Goiânia, UFG, 1988, 155 p. (Tese mestrado)

EBERHART, S.A.; RUSSELL, W.A. Stability parameters for comparing varieties. Crop Science, v. 6, p. 36-40, 1966.

FINLAY, K.W.; WILKINSON, G.N. The analysis of adaptation in a plant breeding programme. Australian Journal of Agricultural Research, Melbourne, v. 14, n. 6, p. 742-754, 1963.

HAMASAKI, R.I.; BRAZ, L.T.; PURQUERIO. L.F.V.; PEIXOTO, N. Comportamento de novas cultivares de feijão-vagem em Jaboticabal-SP. CONGRESSO BRASILEIRO DE OLERICULTURA, 38., 1998, Petrolina. Resumo... Petrolina: SOB, 1998.

PEIXOTO, N.; SILVA, L.O.; THUNG, M.D.T.; SANTOS, G. Produção de sementes de linhagens e cultivares arbustivas de feijão-vagem em Anápolis. Horticultura Brasileira, Brasília, v. 11, n. 2, p. 151-152, 1993. 